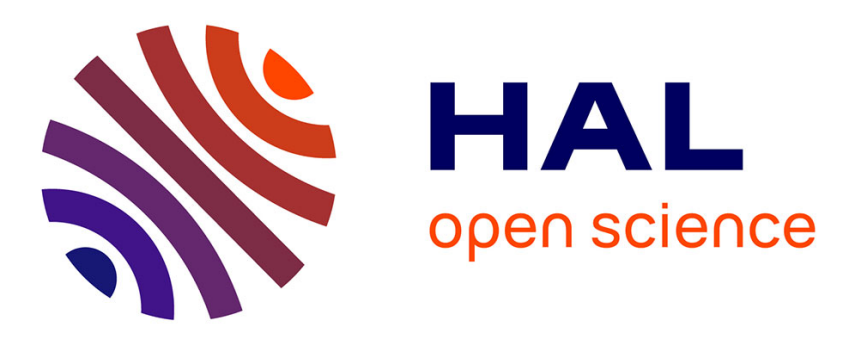

\title{
Motifs in regulatory networks and their structural robustness
}

\author{
Adrien Elena, Hedi Ben-Amor, Nicolas Glade, Jacques Demongeot
}

\section{To cite this version:}

Adrien Elena, Hedi Ben-Amor, Nicolas Glade, Jacques Demongeot. Motifs in regulatory networks and their structural robustness. 8th IEEE International Conference on BioInformatics and BioEngineering, 2008. BIBE 2008., Oct 2008, Athens, Greece. pp.1-6, 10.1109/BIBE.2008.4696699 . hal-00345993

\section{HAL Id: hal-00345993 \\ https://hal.science/hal-00345993}

Submitted on 10 Dec 2008

HAL is a multi-disciplinary open access archive for the deposit and dissemination of scientific research documents, whether they are published or not. The documents may come from teaching and research institutions in France or abroad, or from public or private research centers.
L'archive ouverte pluridisciplinaire HAL, est destinée au dépôt et à la diffusion de documents scientifiques de niveau recherche, publiés ou non, émanant des établissements d'enseignement et de recherche français ou étrangers, des laboratoires publics ou privés. 


\title{
Motifs in regulatory networks and their structural robustness
}

\author{
A. Elena, H. Ben-Amor, N. Glade \& J. Demongeot \\ TIMC-IMAG, UMR CNRS 5525 \\ Faculty of Medicine of Grenoble \\ University J. Fourier \\ $38700 \quad$ La Tronche France \\ E-mail: Hedi.Ben-Amor@imag.fr, Adrien.Elena@imag.fr, \\ Nicolas.Glade@imag.fr, Jacques.Demongeot@imag.fr
}

\begin{abstract}
If we consider the regulatory networks used in systems biology (especially in neural and genetic networks), we observe certain regularities in their architecture, namely the occurrence of interaction motifs, i.e. of oriented sub-graphs relating their elements with the same interaction scheme. We propose here to describe some of these motifs we can consider as universal because they are often met in the regulatory networks architecture, and to study their main robustness properties (called also structural stability), i.e. their ability to keep after topological or dynamical perturbations, their essential asymptotic features, as their attractors number, shape and behaviour, like their periodicity.
\end{abstract}

Keywords: regulatory networks, interaction motifs, robustness, structural stability, attractors, periodicity

\section{Introduction}

The regulatory networks (neural, genetic, ...) are widely used in systems biology and they present some remarkable regularities in their architecture, namely the same values for architectural (connectivity coefficient or number of connex components) or dynamic (number of attractors or stability basins size) features or occurrence of same interaction motifs, i.e. oriented sub-graphs relating their elements (neurons, genes,...) into the same interaction schemes.

These similarities cause identical dynamical behaviours, like periodic attractors, due for example to the same internal motif imposing its periodicity to the global network. The interaction matrix $\mathrm{W}$ of a regulatory network is the oriented incident matrix of the interaction graph of the network. It can be either estimated from the observation of co-occurrence of neural or genic activities (which allows to calculate the empirical auto-correlation matrix of activities) or calculated from the discrete or continuous Jacobian matrix $J$ of a mathematical model proposed for describing the biological mechanisms underlying the regulation process. In particular, $\mathrm{W}$ and $\mathrm{J}$ are sharing the same associated interaction graph and $\mathrm{W}$ can be considered as the signed version of $\mathrm{J}$. W is then similar to the synaptic weights matrix, which rules the relationships between neurons in a neural network. The general coefficient $\mathrm{w}_{\mathrm{ik}}$ of such a matrix W is equal to +1 (resp. $-1,0$ ) if the gene (or the neuron; in the sequel, we will choose both genetic and neuronal examples) $G_{k}$ activates (resp. inhibits, does not influence) the gene $G_{i}$, the state $x_{i}$ of the gene $G_{i}$ being equal to +1 (resp. 0 ), if it is (resp. is not) expressed. In the case of small regulatory genetic networks (called operons), the knowledge of $\mathrm{W}$ permits to make explicit all possible stationary behaviours (fixed or cyclic configurations of genes expression states) of any organism having the corresponding genome. The change of state of $\mathrm{G}_{\mathrm{i}}$ between times $t$ and $t+1$ obeys a threshold rule: $\mathrm{x}_{\mathrm{i}}(\mathrm{t}+1)=\mathrm{H}\left(\sum_{\mathrm{k}=1, \mathrm{n}} \mathrm{w}_{\mathrm{ik}} \mathrm{x}_{\mathrm{k}}(\mathrm{t})-\theta_{\mathrm{i}}\right)$ or $\mathrm{x}(\mathrm{t}+1)=\mathrm{H}(\mathrm{Wx}(\mathrm{t})-\theta)$, where $\mathrm{H}$ is the sign step function $(\mathrm{H}(\mathrm{y})=1$, if $\mathrm{y}>0$ and $\mathrm{H}(\mathrm{y})=0$, if $\mathrm{y} \leq 0)$ and the $\theta_{\mathrm{i}}$ 's are threshold values. When $\mathrm{t}$ is increasing, the genes states reach a set of configurations (fixed or cyclic) called attractor of the genetic network dynamics and do not change any more. It is in general of a great biological interest to determine the interaction matrices having characteristic properties like i) a minimal number of non zero coefficients for a given set of attractors or ii) a minimal number $\mathrm{P}(\mathrm{W})$ of positive loops (i.e. paths on the interaction graph coming from a gene and returning to it after an even number of negative interactions, symbolized by negative edges), controlling the number $\mathrm{A}(\mathrm{W})$ of attractors (cf. for details Demongeot et al., 2000a; 2000b; Cinquin and Demongeot, 2002a; 2002b; Soulé, 2003; Aracena et al., 2004a, b, c, d; Soulé et al., 2007; Elena and Demongeot, 2008). The connectivity coefficient $\mathrm{K}(\mathrm{W})=\mathrm{I} / \mathrm{n}$, is the ratio between the number $\mathrm{I}$ of interactions and the number $\mathrm{n}$ of genes (the dimension of $\mathrm{W}$ is $\mathrm{n} . \mathrm{n}$ ), and the mean inhibition weight $\mathrm{I}(\mathrm{W})=\mathrm{R} / \mathrm{I}$, is the ratio between the number of inhibitions (or repressions) $\mathrm{R}$ and $\mathrm{I} . \mathrm{K}(\mathrm{W})$ is in general between 1.5 and 3 and $\mathrm{I}(\mathrm{W})$ between $1 / 3$ and $2 / 3$, both in neural and genetic regulatory networks. The number of connex components (i.e. with a path between each pair of nodes) containing positive loops, denoted $\mathrm{C}(\mathrm{W})$, verifies the following conjectures:

$$
2^{\mathrm{P}(\mathrm{W})} \geq \mathrm{F}(\mathrm{W}) \geq 2^{\mathrm{C}(\mathrm{W})} \text { and } \mathrm{F}(\mathrm{W})=\mathrm{O}\left(\mathrm{n}^{1 / 2}\right),
$$

where $\mathrm{F}(\mathrm{W})$ is the number of fixed configurations, the total number of attractors being of greater order of magnitude, due to the presence of numerous limit cycles (Kauffman et al., 2003; 2004). In the case of large regulatory networks now frequently studied (Karlsson and Hörnquist, 2007), their study needs in general an approach using huge simulations (e.g. for showing the existence of phase transitions, like in Demongeot et al., 2008 and Demongeot and Sené, 2008), hence we will focus here on the extraction of characteristic sub-networks with common features called motifs, from the whole regulatory network and we will try to deduce some general rules coming from the systematic study of the asymptotic dynamical behaviour of such motifs.

\section{Description of the interaction motifs}

In many regulatory networks like the interaction graph which rules the spatial stripes morphogenesis in Drosophila melanogaster (Ishihara et al., 2005), we can extract motifs like the mixed forward inhibition/activation one, called incoherent feed-forward loop in (Alon, 2007).

Frequent motifs extracted from regulatory networks are given in 
Figure 1: incoherent feed-forward loop (1), triple negative loop (2), 3 -switch (3), negative (4) and positive (5) regulons. We can remark that they are pauci-genic (at most 5 genes) and located in the core (Elena and Demongeot, 2008) of the networks from which they have been extracted. We will in the following give general statements available for such pauci-genic motifs.
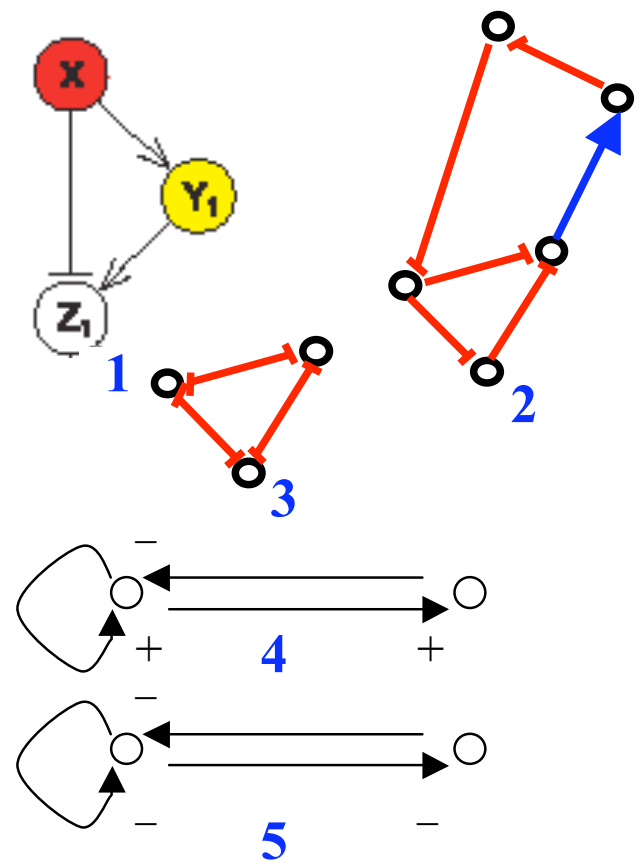

Figure 1. Motifs extracted from regulatory networks

\section{Dynamics of the motifs}

\subsection{Standard updating methods}

Let us suppose that the network is made of $n$ vertices whose dynamical rule of state transition is of the Hopfield type, i.e. if $x_{i}(t)$ denotes the state ( 1 if the node $i$ is activated, and 0 elsewhere), we have: $\quad \mathrm{x}_{\mathrm{i}}(\mathrm{t}+1)=\mathrm{H}\left(\Sigma_{\mathrm{j}=1, \ldots, \mathrm{n}} \mathrm{w}_{\mathrm{i}, \mathrm{j}} \mathrm{x}_{\mathrm{i}}(\mathrm{t})-\theta_{\mathrm{i}}\right)$, where $\mathrm{H}$ denotes the Heaviside function $(\mathrm{H}(\mathrm{y})=1$, if $\mathrm{y}>0 ; \mathrm{H}(\mathrm{y})=0$, elsewhere), the $\mathrm{w}_{\mathrm{i}, \mathrm{j}}$ 's are the interaction weights (with $\mathrm{w}_{\mathrm{i}, \mathrm{j}}<0$, if $\mathrm{j}$ inactivates $\mathrm{i} ; \mathrm{w}_{\mathrm{i}, \mathrm{j}}$ $>0$, if $j$ activates $i$ and $w_{i, j}=0$, if $j$ has no influence on i) and $\theta_{i}$ is a threshold. Based on this updating rule, we can define three types of updating methods:

- sequential iterations, where the states of the nodes are sequentially updated,

- parallel iteration, where the states of all the nodes are synchronously updated,

- block-sequential iterations, where the nodes are grouped into ordered blocks (one node can be in only one block). The blocks are sequentially updated, and inside a block nodes are synchronously updated.

We can notice that sequential iterations and parallel iterations are particular cases of block-sequential iterations.

The state of the entire network is considered only after all nodes have been updated. The dynamics of the network, and especially its attractors depend on the updating method. For the 3-switch (case 3 of Figure 1) for example where all $\mathrm{w}_{\mathrm{i}, \mathrm{j}}$ 's are negative (supposed in the following equal to -1 , all thresholds being equal to 0 ), we have: - for the sequential iterations, the system has only 2 fixed configurations, with state 1 (resp. 0 ) at the first visited node and state 0 (resp. 1) at the other nodes, these values depending on the chosen sequential updating order. Such a system having only fixed configurations is potential in the sense of (Demongeot et al., 2006b), because the discrete velocity of the dynamics is equal to the gradient of a Lyapunov function (it is for example the case in a $\mathrm{n}$-switch when the interaction weights are symmetrical)

- for the parallel iterations, we have a cycle of order 2 (made of the full 0 and full 1 configurations), and 6 fixed configurations (the same fixed points as in sequential update). Such a system is the equivalent of a Hamiltonian continuous dynamical system without attraction basins, and it will be called Hamiltonian network.

- for the block-sequential iterations, we have the same fixed configurations as in sequential iterations. From this particular network 3-switch, we conjecture that the cyclic behaviour of the networks of size 3 can appear when the updating method goes from sequential to the parallel iterations and not in the inverse way. The statistics done on the set of the 188968 networks of size 3 show that this conjecture is almost everywhere true, but it fails for $0.3 \%$ of them. Certain other observations can be generalized:

Lemma 1 When a cycle occurs in the dynamics of a block, then the whole network dynamics has only cycles as attractors. Reciprocally, if the global network dynamics has a cycle, there is necessary at least one block having at least a cycle as attractor.

Lemma 2 If the global network dynamics has a cycle of length $\mathrm{m}$, then $\mathrm{m} \leq \Pi_{\mathrm{i} \in \mathrm{C}} \mathrm{m}_{\mathrm{i}}$, where $\mathrm{C}$ is the set of the blocks having at least a cycle as attractor, $m_{i}$ being the maximal length of cycles of block $i$.

The proof of the 2 Lemmas above is obvious. The statistical behaviours observed for the size 3 networks need an exhaustive simulation of their dynamics; we are interested here in size 3 networks having some common dynamical features: for certain updating methods, they have both fixed configurations and at least one limit cycle, the limit cycles disappearing completely for other iteration rules, where there are only fixed configurations. Among the 188968 possible networks of size 3 , only $34947(18,5 \%)$ have this dynamical behaviour. If we try to understand how the limit cycles are disappearing when we change of iteration method, we observe that frequently this disappearance occurs when we go from the parallel iteration to the sequential ones. As mentioned previously sequential and parallel iterations are particular cases of block-sequential iterations. We define a hierarchy of blocksequential iterations. An iteration mode $\mathrm{A}$ is a child in this hierarchy of an iteration mode B (B is then the "parent" of A), if A has one block more than $\mathrm{B}$ and if we can obtain $\mathrm{B}$ by bringing together 2 consecutive blocks of A. The parallel iteration is the root of the hierarchy while sequential modes are the tips. We can then study the way in which the limit cycles may occur or disappear at a given level of the hierarchy. We observe in the simulations three different possible behaviours:

- networks with limit cycles disappearing when going down in the hierarchy from the root to the tips ("Down" behaviour)

- networks with limit cycles disappearing when going up in the hierarchy from the tips to the root ("Up" behaviour)

- networks that are not corresponding to any previous behaviour, for which the limit cycles occur and disappear inside the hierarchy without clear rule ("None" behaviour).

Among the 34947 simulated networks of size 3 having limit cycles and fixed points for some iterations modes and only fixed points for others, the dispatching follows the repartition below:

\begin{tabular}{|c|c|c|c|}
\hline Down & None & Up & Total \\
\hline 21729 & 13110 & 108 & 34947 \\
\hline $62,18 \%$ & $37,51 \%$ & $0,31 \%$ & $100 \%$ \\
\hline
\end{tabular}

This repartition confirms that there is practically no network (only $0.31 \%$ ) for which the cycles are present in the sequential updating modes and disappear in the synchronous one. A general result for Boolean networks has been recently proved in (Goles and Salinas, 2008) : a limit cycle for parallel iteration is never a limit cycle for any sequential iteration. 


\subsection{An other way to update the state of the network}

From a biological point of view the main issue of the updating methods presented in the previous part is that we consider the state of the network as updated only after all the states of the entire set of nodes have been updated. Practically, if we observe in experiments the expression of genes evolving in time, how can we know when the expression of all genes has been updated ? For this reason we introduce an other way to update the state of the network. The updating methods presented in part 3.1 remain the same. But now we consider the state of the network after the updating of each block of nodes (each node in the case of sequential iterations). It is obvious that for the parallel iteration there is no change. For the other updating methods, there are k-1 (where $\mathrm{k}$ is the number of blocks ; $\mathrm{k}=\mathrm{n}$ for sequential iterations) new network's states between two consecutive states of the standard updating methods. With this new way to update the state of the network, the last result of part 3.1 is no longer true. Let us take the example of the positive regulon introduced in Figure 1. The graph of this motif is shown in Figure 2 with the corresponding interaction matrix $\mathrm{W}$ and threshold vector $\theta$. With the standard updating way, there is a limit cycle of length 4 in the parallel iteration and a limit cycle of length 2 in both sequential iterations. But with this new way of updating the network's state, the regulon reaches the same limit cycle of length 4 whatever the iteration mode is and then there is a limit cycle $\mathrm{C}$ for the parallel iteration that is also a limit cycle $\mathrm{C}$ for the sequential iterations.

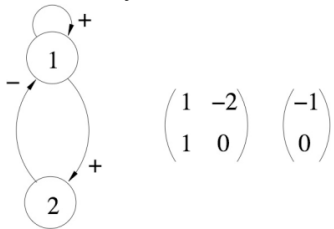

Figure 2. Graph of the positive regulon with the corresponding $\mathrm{W}$ and $\theta$.

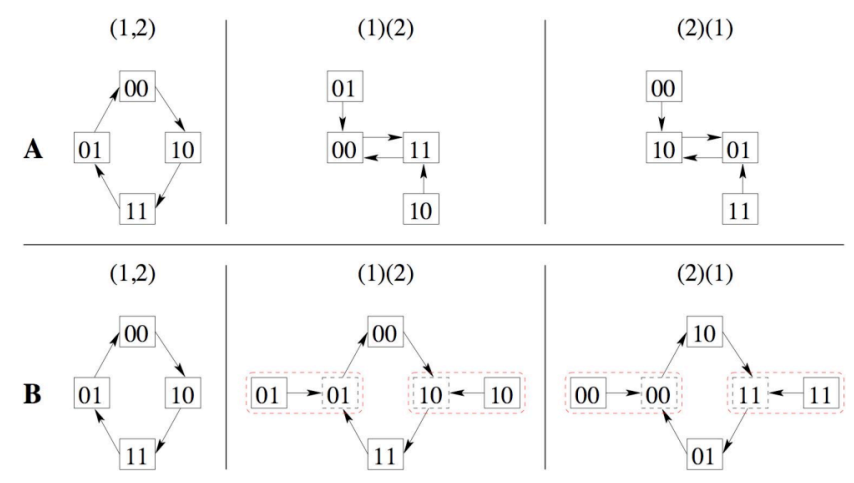

Figure 3. Iteration graphs for the positive regulon of Figure 2 A) corresponding to the standard way of updating for the parallel (left) and sequential iterations (centre and right) and B) to the new way of updating (left to right same as in A). Transitions that have been added in the new way of updating are represented with dotted lines.

We can notice that if we observe the evolution of the gene expression in a positive regulon, there is no way to distinguish the updating methods that is underneath the dynamics. The iteration graphs corresponding to the newly introduced way of updating (that fits what we can observe in practical) are indeed the same for all updating methods (Figure $3 \mathrm{~B}$ ). A third theoretical result can be proved:

Lemma 3 Let $\mathrm{N}$ be a network of size $\mathrm{n}$ with limit cycles for parallel and sequential iterations (with the standard updating). If there is no couple of limit cycles $\left(\mathrm{C}_{1}, \mathrm{C}_{2}\right)$ of lengths $\left(\mathrm{l}_{1}, \mathrm{l}_{2}\right)$ where $\mathrm{C}_{1}$ is a limit cycle for the parallel iteration and $\mathrm{C}_{2}$ for the sequential one with $1_{1}=\mathrm{n} * 1_{2}$, there is no limit cycle reached both for parallel and sequential iterations with the new updating.
The proof of this lemma is obvious by considering the number of states that are added with the newly introduced way of updating for a sequential iteration. The negative regulon (Figure 4) gives a good example of network (or motif) where there is no limit cycle shared by the parallel iteration and a sequential iteration. As shown in Figure 5 with the standard way of updating, there is a unique limit cycle of length 3 for the parallel iteration and a unique limit cycle of length 2 for both sequential iterations.

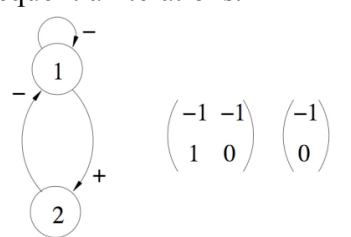

Figure 4. Graph of the negative regulon with the corresponding $\mathrm{W}$ and $\theta$.

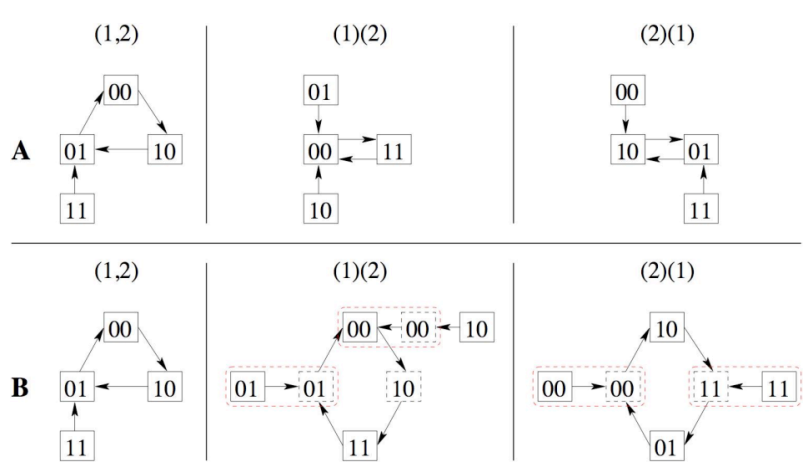

Figure 5. Iteration graphs for the negative regulon of the Figure 4 (same description as in Figure 3).

The last parts show the importance of studying the robustness of networks dynamics to the updating methods.

\section{Robustness to global statistical topology perturbations}

Certain perturbations can globally affect or not the dynamical behaviour of the regulatory networks. For example, an environmental noise can both change the number of nodes synchronously updated in a block (like in the previous Section) or the network topology by keeping or cancelling certain vertices or edges of the network. By simulating two very simple toy networks, the game of life and the cardio-respiratory coupling, we will study in the following how and where act such perturbations. We have developed an integrated software for designing networks of various nature (neuronal, regulatory, metabolic, social,...), allowing the simulations of these different kinds of networks and their analyse (notably the program gives the global activity of the network and can return the shape of the attractors and of the attraction basins for all the networks corresponding to a given structure. The software is constructed around a core which is independent of the nature of the network. This core includes a list of nodes and links. The latter are themselves composing structures that can act as buffers during the information transfer as it is the case for the electric depolarising waves that propagate along the axons. This is useful for imposing delays in the information propagation as a physical representation of distances between nodes. The core also includes an update scheduling plan that allows assigning different priorities in the update of some parts of the network. Finally, the software uses a graphical interface for creating and parametrizing the networks and for launching simulations and following the running process. We used this tool so as to study the effect of stochasticity and of synchronicity on the robustness of our networks. Stochasticity takes place in two components of the network. First, it is applied within the vertices in the form of a temperature parameter, notably 
in the Boltzmann equation that describes the update of the target nodes. This represents the manner the connexions make sometimes errors, or in the extreme case behave independently of their entries. Moreover, we allow the single nodes to stochastically switch within the panel of states they can take. Single nodes in a certain state can indeed change to another state given a transition law. This law can be purely random but we preferred using a particle diffusion model. A certain diffusivity constant set can be then assigned to each node so as to describe the probabilities of transition between the possible reachable states of the node.

\subsection{A first toy model, the game of life}

The game of life is a very famous cellular automaton devised in (Gardner, 1970) and proved to be computation universal (Durand and Roka, 1998). In (Fates and Morvan, 2004), the game of life is implemented in a 2D cellular automata run on a regular subset of $Z^{2}$ where the neighbourhood of each cell is the Moore one (i.e. the 8 nearest neighbours). It has been shown in (Blok and Bergersen, 1997) that the role of this type of boundary conditions was important concerning the dynamical behaviour of the game of life. Here, we consider a finite grid of $Z^{2}$. At each time step $t$, the following effects occur: (i) any living cell with fewer than 2 living neighbours dies, as if by loneliness, (ii) any living cell with more than 3 living neighbours dies, as if by overcrowding, (iii) any living cell with 2 or 3 living neighbours lives and (iv) any dead cell with exactly 3 neighbours comes to life. The game of life is shown to be unstable against synchrony variation obtained by introducing the probability $\alpha$ for a vertex to be updated at any time $t$ as shown in Figure 6, which highlights a labyrinthic structure (not realistic concerning its "living" character) when the synchrony rate $\alpha$ decreases. However, it is possible to increase the robustness to asynchrony of the game of life by perturbing the topology of the network. The choice made here is to balance the instability due to an updating property, the decrease of the synchrony rate, with a structural property, which is here the definitive removal of a percentage of edges. This process is of great biological interest because the aim of this work is to know how an already structurally perturbed system reacts against synchrony variations.

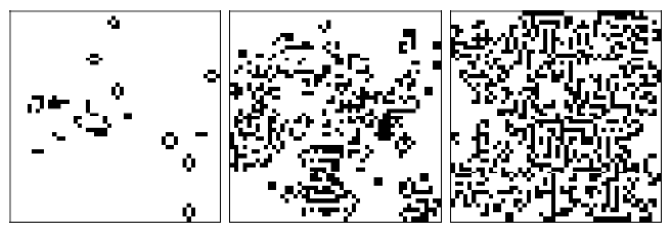

Figure 6. Simulation of the evolution of the game of life, for different synchrony rates: $\alpha=1.0$ (left), $\alpha=0.75$ (centre) and $\alpha=0.5$ (right).

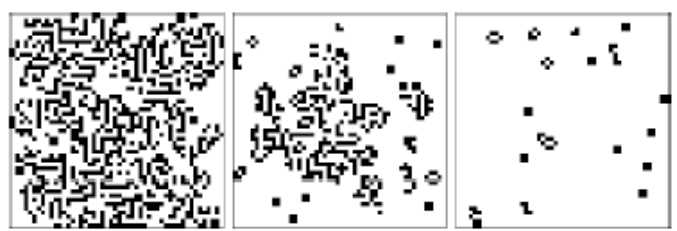

Figure 7. Simulation of the increasing of the robustness to asynchrony of the game of life by perturbing, when the synchrony rate is $\alpha=0.5$, the topology with different probabilities of edge cancelling: $\beta=0$ (left), $\beta=$ 0.05 (centre) and $\beta=0.1$ (right).

Let $\mathrm{G}=(\mathrm{V}, \mathrm{E})$ be the oriented graph of interactions: $\left(\mathrm{v}, \mathrm{v}^{\prime}\right)$ is an edge of $\mathrm{E}$, if and only if $\mathrm{v}^{\prime}$ is in the Moore neighbourhood of $\mathrm{v}$. The perturbed graph $\mathrm{G}^{\prime}=\left(\mathrm{V}, \mathrm{E}^{\prime}\right)$ is obtained by executing the following procedure: for each cell $\mathrm{v}$ in $\mathrm{V}$, for any cell $\mathrm{v}^{\prime}$ in the Moore neighbourhood of $\mathrm{v}$, the edge $\left(\mathrm{v}, \mathrm{v}^{\prime}\right)$ is removed with the probability $\beta$. This probability is called the probability of edge cancelling or the missing-link rate. The results of this study can be seen in the Figure 7, which shows that the increase of the missing-link rate makes the game of life stable against the decrease of the synchrony rate, for which it is non robust (cf. Figure 6). The behaviour of the game of life keeps its normal properties when the synchrony decreases, and its configurations do not enter in a labyrinthic phase. We can easily link this study with the genetic regulatory networks because, if we want to render cellular automata closer to real biological networks, it is necessary to break their regularity by perturbing edges in their interaction graph.

\subsection{A second toy-model, the coupling between the cardiac} and respiratory systems

The functional coupling existing between heart and lungs is a well known example of coupled oscillators. Both organs possess indeed their own activity. The heart beats at about $1 \mathrm{~Hz}$ due to the self-stimulation of some of its specialized cells, those of the sinusal node (or S). These self-rhythmic cells emit periodically action potentials that are transmitted from their origin, the sinusal node, to all the cells by the intermediate of the His fascicle that terminates with Purkinje fibres. Then the electric depolarization is transmitted from a muscular cell to another muscular cell throughout the whole heart muscle. Moreover, a group of selfexcitable neurons present in the cephalo-rachidian bulb, called cardiomodulator neurons (or $\mathrm{C}$ ), exert a control on the rhythmic activity of the sinusal node. Inversely, when firing, the sinusal node cells repress C. In an idealised manner, this can be modelized in the form of an anharmonic oscillator such as a van der Pol system as described in (Glade et al, 2007; Forest et al, 2007). We have shown in (Demongeot et al, 2007a \& 2007b) that such systems are Liénard systems and that they can be decomposed into their Hamiltonian and Potential parts. In the same manner, this can be also done for describing the inspiratory-expiratory cycle of the lungs that has a period of about $4 \mathrm{~s}$ (frequency $0.25 \mathrm{~Hz}$ ). The inspiratory neurons (I) fire from a self-excitable vegetative bulbar center synchronously with the phrenic nerve. On the contrary, the expiratory neurons (E) fire during the silence of the phrenic nerve. $\mathrm{E}$ is activated by I (via the pleural stretch receptors) and $\mathrm{E}$ inhibits I (through intra-bulbar connections). Let consider 2 coupled van der Pol differential equations describing this dynamical behaviour: $\mathrm{dx} / \mathrm{dt}=\mathrm{y}, \mathrm{dy} / \mathrm{dt}=-\mathrm{x}+\varepsilon\left(1-\mathrm{x}^{2}\right) \mathrm{y}$ (respiratory system, where $\mathrm{x}$ represents the activity of neurons $E$, $y$ the activity of neurons $I$, and $\varepsilon$ is the anharmonic parameter, and $\mathrm{dz} / \mathrm{dt}=\mathrm{w}, \mathrm{dw} / \mathrm{dt}=-\mathrm{z}+\eta\left(1-\mathrm{z}^{2}\right) \mathrm{w}+\mathrm{k}(\mathrm{y}) \mathrm{y}$ (cardiac system, where $\mathrm{z}$ represents the activity of neurons $\mathrm{S}, \mathrm{w}$ the activity of neurons $\mathrm{C}, \eta$ is the anharmonic parameter, and $\mathrm{k}(\mathrm{y})$ is the coupling intensity parameter between the inspiratory neurons I and the cardiomodulator $\mathrm{C}$ ). Both organs have their own rhythm but they are also coupled directionally: $\mathrm{C}$ is coupled to the activity of the lungs (inspiratory neurons) via bulbar connections.
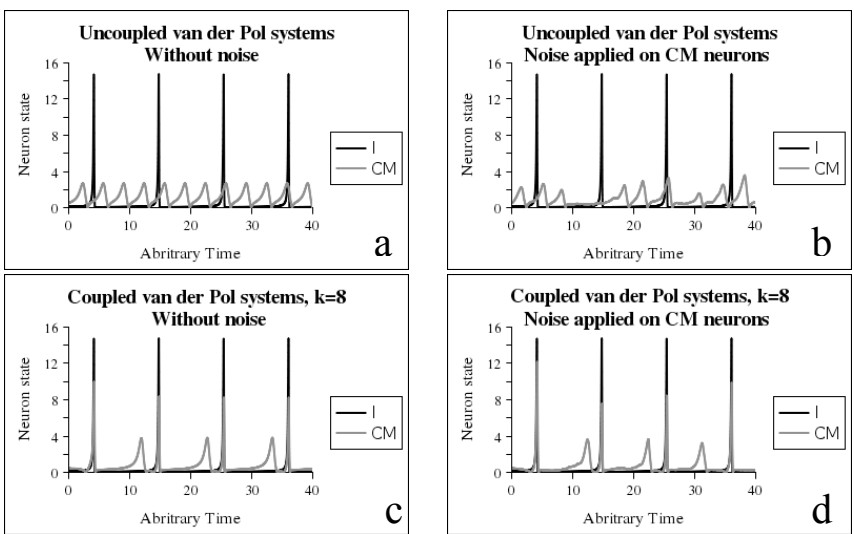

Figure 8. Temporal series obtained by simulating 2 van der Pol systems coupled on their self-excitable nodes. We follow here the activity of these two nodes, the first one for the respiratory system (neurons I in black) and the other for the cardiac system (C neurons in grey), in 4 cases: a) when the 2 van der Pol systems are uncoupled and regular ; b) uncoupled with addition of noise to S neurons; c) coupled together and regular; d) coupled with addition of noise. Parameters are $\varepsilon=10, \eta=1$, with $k(y)=0$ when systems are uncoupled and $\mathrm{k}(\mathrm{y})=8$ when coupled. 
This allows the bulbar vegetative control system to adapt to the effort: first the breathing is entrained by a muscular activity and secondarily entrains the heart. Such a capacity of adaptation disappears in degenerative diseases like Parkinson or diabetes. Two kinds of dysfunctions can be studied with our coupled oscillators : those that affect the coupling $\mathrm{k}(\mathrm{y})$ between the two oscillators and those that affect the periodicity of the heart. During myocardial infarction and ischemia, local cell deaths in the heart tissue can occur causing local loss of the electric signal initiated in the sinusal node and the His fascicle. This manifests in the form of spiral cardiac reentries and ventricular fibrillation (Bardou et al, 1995). Nevertheless, the forcing induced by the vegetative control of the respiratory system can correct in a limited manner this arrhythmia. As a consequence, a loss of the entrainment of the heart beating period by the respiratory system avoids any recuperation of an acceptable periodicity in case of heart arrhythmias. These behaviours are illustrated with two coupled van der Pol systems in Figure 8 . The vegetative control of the cardio-respiratory system can also be described by a more simple scheme where both cardiac and respiratory systems are represented by a Hopfield-like network. Two regulons (with $\mathrm{S}$ and $\mathrm{C}$ as sinusal node and cardiomodulator node, and I and $\mathrm{E}$ as inspiratory and expiratory nodes) are coupled between the inspiratory node of respiratory system and the cardiomodulator (Figure 9 b). Each negative regulon (Figure 9 a) is composed of two nodes N0 as I (resp. C) and N1 as E (resp. $\mathrm{S}$ ), where the nodes N0 (I or C) are the self-regulated nodes.
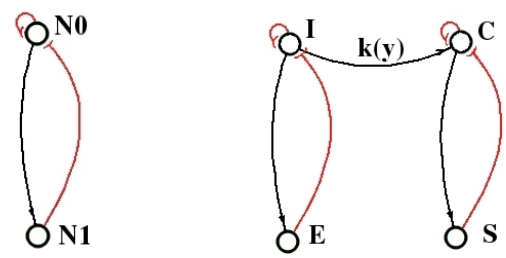

Figure 9. Structure of a single negative regulon (left) with its two nodes, N0 the self-excitable one and N1. On the right are shown two regulons coupled between their respective self-excitable nodes $\mathrm{I}$ and $\mathrm{C}$ by a directional edge with a coupling intensity $\mathrm{k}(\mathrm{y})$. In this toy model, the first regulon represents the vegetative control of the respiratory system with its inspiratory (I) and expiratory (E) neurons ; the second one describes the vegetative control of the cardiac oscillator with the cardiomodulator bulbar node $(\mathrm{C})$ regulating the activity of the sinusal node $(\mathrm{S})$.

The stochastic transition rule used is:

$\mathrm{P}\left(\mathrm{x}_{\mathrm{i}}(\mathrm{t}+1)=1 \mid \mathrm{x}_{\mathrm{k}}(\mathrm{t}), \mathrm{k}=1, \mathrm{n}\right)=\exp (\mathrm{H}(\mathrm{t})) /(1+\exp (\mathrm{H}(\mathrm{t})))$, where $\left.\mathrm{H}(\mathrm{t})=\sum_{\mathrm{k}=1, \mathrm{n}} \mathrm{w}_{\mathrm{ik}} \mathrm{x}_{\mathrm{k}}(\mathrm{t})-\theta_{\mathrm{i}}\right) / \mathrm{T}$, with:

Interaction matrix W

\begin{tabular}{|c|c|c|}
\cline { 2 - 3 } Node & N0 & N1 \\
\hline N0 & -1 & -1 \\
\hline N1 & 1 & 0 \\
\hline
\end{tabular}

Node thresholds $\theta$

\begin{tabular}{|c|c|}
\hline Node & Threshold \\
\hline N0 & $-1,5$ \\
\hline N1 & 0 \\
\hline
\end{tabular}

Between the nodes N0 (I and C) of the two regulons we have an interaction with positive weight called $\mathrm{k}(\mathrm{y})$. Then our network is composed of 2 oscillators connected by a positive interaction. The frequency of the cardiac system is faster than that of the respiratory system. In the Hopfield network this can be clearly expressed by using a specific update schedule. The simplest one is block sequential. It is made of 9 blocks B0, B1, ..., B8. Those blocks are ordered as follow and allow obtaining frequencies of oscillation 2 to 4 times faster (depending on their contents, here 3 times) for the cardiac system than for the respiratory system :

\begin{tabular}{|l|l|l|l|l|l|l|l|l|l|}
\hline $\begin{array}{l}\text { Update } \\
\text { Block }\end{array}$ & B0 & B1 & B2 & B3 & B4 & B5 & B6 & B7 & B8 \\
\hline $\begin{array}{l}\text { Node } \\
\text { Content }\end{array}$ & $\begin{array}{l}\text { I, E, } \\
\text { C,S }\end{array}$ & $\begin{array}{l}\text { I, E, } \\
\text { C,S }\end{array}$ & $\begin{array}{l}\text { I, E, } \\
\text { C,S }\end{array}$ & C,S & C,S & C,S & C,S & C,S & C,S \\
\hline
\end{tabular}

We show here that it is easy to obtain the same kind of behaviour than with a differential equation system, but only with boolean dynamics. Introducing stochasticity on the cardiac sinusal node allows simulating a heart arrhythmia, and we also verify that by reducing or removing the coupling between I and C nodes we loose the period entrainment and then a corrective functionality in case of heart arrhythmia. This is illustrated in Figure 10.
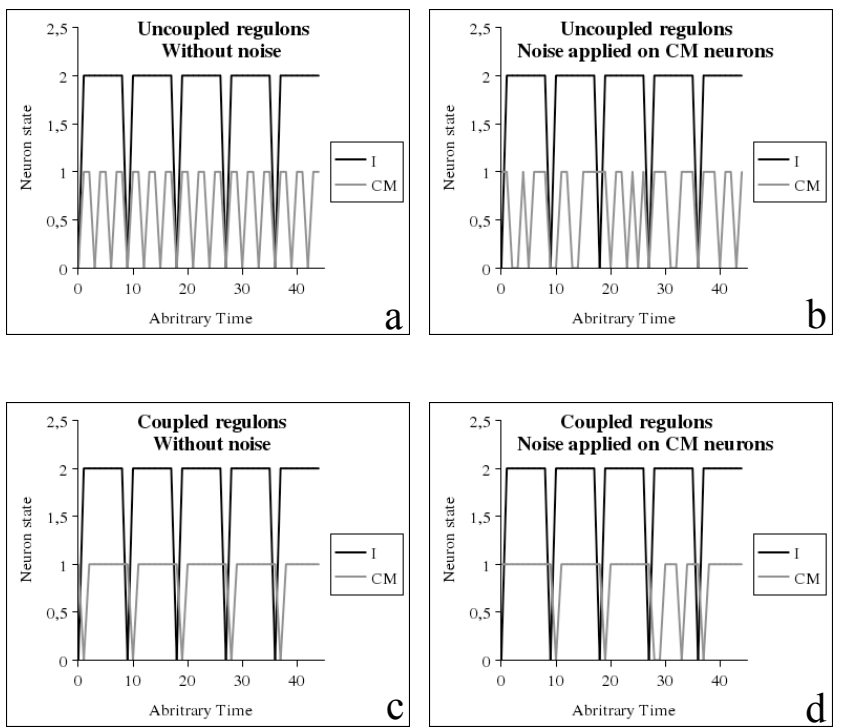

Figure 10. Temporal series from simulations of two Hopfield networks (negative regulons) coupled between their self-excitable nodes. As on Figure 8 , we follow the activity of the 2 nodes (neurons I in black and $\mathrm{C}$ in grey), in 4 cases : regulons are uncoupled and regular (top left) ; uncoupled with addition of noise to $\mathrm{S}$ neurons (top right); coupled together and regular (bottom left); coupled with addition of noise (bottom right).

We first suppose the 2 oscillators as independent. We clearly observe that the beating frequency of the heart regulon is 3 times faster than the breathing frequency. When a coupling is added $(\mathrm{k}(\mathrm{y})=5)$, the respiratory system rhythm forces the cardiac system to enter in resonance with itself. Then we suppose the heart as sick, so we introduce an important noise on the heart system regulon. To perform this we use a stochastic updating rule in node NO (C) and N1 (S) with a temperature equal to 1.5 . As previously we observe the 2 following subcases : if the systems are uncoupled, beatings of heart are no longer regular even if breathing is healthy. However, when coupled $(\mathrm{k}(\mathrm{y})=5)$, despite the fact that there is an important noise in heart beating, the respiratory system allows maintaining a periodic oscillation on the targeted regulon by forcing it (the cardiac system). Here, the robustness is obtained by imposing a strong activity of regulation on the noisy part of the network.

\section{Conclusion}

We have shown in this paper that a complex regulatory network contains in general characteristic sub-networks called motifs whose dynamics is easer to be simulated or theoretically predicted than the global one. If these motifs have a periodic behaviour, they impose their periodicity to the global network dynamics. This 
behaviour can be robust or not and we have given some indications about two types of robustness, one related to changes in motifs architecture due to a local deterministic perturbation (like a gene know-out killing the expression of a repressor protein, whose cancelling restores the repressed node in the network) and the other related to statistical topology perturbations due for example to an environmental noise. Both types of perturbations can cause the disappearance or the conservation of dynamical patterns like limit cycles and we have given some indications on the way to reach robust dynamical behaviours. General statements are very difficult to be theoretically proven, but certain statistical regularities can be shown by simulation.

Acknowledgements We are indebted to L. Glass for helpful discussions and comments.

\section{References}

Alon, U. (2007) 'Network motifs: theory and experimental approaches', Nature Rev. Genetics Vol. 8, pp. 450-461.

Aracena, J., Ben Lamine, S., Mermet, M.A., Cohen, O. and Demongeot, J. (2003) 'Mathematical modelling in genetic networks: relationships between the genetic expression and both chromosomic breakage and positive circuits', IEEE Trans. Systems Man Cyber., Vol. 33, pp. 825-834.

Aracena, J., Demongeot, J. and Goles, E. (2004a) 'Mathematical modelling in genetic networks', IEEE Trans. Neural Networks, Vol. 15, pp. 77-83.

Aracena, J., Demongeot, J. and Goles, E. (2004b) 'Fixed points and maximal independent sets on AND-OR networks', Discr. Appl. Math., Vol. 138, pp. 277-288.

Aracena, J., Demongeot, J. and Goles, E. (2004)c 'On limit cycles of monotone functions with symmetric connection graphs', Theoret. Comp. Sci., Vol. 322, pp. 237-244.

Aracena, J. and Demongeot, J. (2004d) 'Mathematical Methods for Inferring Regulatory Networks Interactions: Application to Genetic Regulation', Acta Biotheoretica, Vol. 52, pp. 391-400.

Aracena, J., Gonzalez, M., Zuniga, A., Mendez, M.A. and Cambiazo, V. (2006) 'Regulatory network for cell shape changes during Drosophila ventral furrow formation', J. Theor. Biol., Vol. 239, pp. 49-62.

Bardou, A.L., Auger, P.M., Achour, S., Dumee, P., Birkui, P.J. and Govaere1, M.-C. (1995) 'Effect of myocardial infarction and ischemia on induction of cardiac reentries and ventricular fibrillation', Acta Biotheoretica Vol. 43, pp. 363-372.

Blok, H.J. and Bergersen, B. (1997) 'Effect of boundary conditions on scaling in the "game of life", Physical Review E, Vol. 55, pp. 6249-6252.

Cinquin, O. and Demongeot, J. (2002a) 'Positive and negative feedback: striking a balance between necessary antagonists', J. Theoret. Biol., Vol. 216, pp. 229-241.

Cinquin, O. and Demongeot, J. (2002b) 'Positive and negative feedback: mending the ways of sloppy systems', C. R. Biologies, Vol. 325, pp. 10851095.

Cinquin, O. and Demongeot, J. (2005) 'High-dimensional switches and the modeling of cellular differentiation', J. Theor. Biol., Vol. 233, pp. 391-411. de Duve, C. (2002) Life Evolving, Oxford University Press, Oxford UK.

Demongeot, J., Aracena, J., Ben Lamine, S., Mermet, M.A. and Cohen, O. (2000a) 'Hot spots in chromosomal breakage: from description to etiology' In: Comparative Genomics, D. Sankoff \& J.H. Nadeau eds., Kluwer, Amsterdam, pp. 71-85.

Demongeot, J., Thomas, R. and Thellier, M. (2000b) 'A mathematical model for storage and recall functions in plants', C. R. Acad. Sc. Sciences de la Vie, Vol. 323, pp. 93-97.

Demongeot, J., Virone, G., Duchêne, F., Benchetrit, G., Hervé, T., Noury, N. and Rialle, V. (2002) 'Multi-sensors acquisition, data fusion, knowledge mining and alarm triggering in health smart homes for elderly people', $C$. R. Biologies, Vol. 325, pp. 673-682.

Demongeot, J., Aracena, J., Thuderoz, F, Baum, T.P. and Cohen, O. (2003a) 'Genetic regulation networks: circuits, regulons and attractors', $C$. R. Biologies, Vol. 326, pp. 171-188.

Demongeot, J., Thuderoz, F., Baum, T.P., Berger, F. and Cohen, O. (2003b) 'Bio-array images processing and genetic networks modelling', $C . R$. Biologies, Vol. 326, pp. 487-500.

Demongeot, J., Thellier, M. and Thomas, R. (2006a) 'Storage and recall of environmental signals in a plant: modelling by use of a differential (continuous) formulation', C. R. Biologies, Vol. 329, pp. 971-978.

Demongeot, J. Elena, A. and Sené, S. (2008) 'Robustness in neural and genetic networks', Acta Biotheoretica, Vol. 56, pp. 27-49.

Demongeot, J., Elena, A. and Weil, G. (2006b) 'Potential automata. Application to the genetic code III', C. R. Biologies, Vol. 329, pp. 953-962. Demongeot, J., Glade, N., Forest, L. (2007a) 'Lienard systems and potential-hamiltonian decomposition. I Algorithm', C. R. Math., Vol. 344, pp. 121-126.

Demongeot, J., Glade, N., Forest, L. (2007b) 'Lienard systems and potential-hamiltonian decomposition. II Methodology', C. R. Math., Vol. 344, pp. 191-194.

Demongeot, J. and Moreira, A. (2007) 'A circular RNA at the origin of life', J. Theor. Biol., Vol. 249, pp. 314-324.

Demongeot, J., Jezequel, C. and Sené, S. (2008) 'Asymptotic behavior and phase transition in regulatory networks. I Theoretical results', Neural Networks, doi:10.1016/j.neunet.2008.04.003.

Demongeot, J. and Sené, S. (2008) 'Asymptotic behavior and phase transition in regulatory networks. II Simulations', Neural Networks, doi:10.1016/j.neunet.2008.04.005.

Durand, B. and Roka, Z. (1998) The game of life: universality, ENS Lyon Research Report \#98-01, Lyon.

Eigen, M. (1971) 'Molekuläre Selbstorganisation und Evolution', Naturwissenschaften, Vol. 58, pp. 465-523.

Eigen, M., Gardiner, W., Schuster, P. and Winkler-Oswatitsch, R. (1981) 'The origin of genetic information' Sci. Am., Vol. 244, pp. 88-92.

Elena, A. and Demongeot, J. (2008) 'Interaction motifs in regulatory networks and structural robustness', In: IEEE CISIS' 08 \& IIBM' 08, IEEE Proceedings, Piscataway, pp. 682-686.

Fates, N. and Morvan, M. (2004) 'Perturbing the topology of the game of life increases its robustness to asynchrony', In: Proceedings of 6th International Conference on Cellular Automata for Research and Industry (ACRI'04), Lecture Notes in Computer Science, Vol. 3305, pp. 111-120.

Forest, L., Glade, N., Demongeot, J. (2007) 'Lienard systems and potentialhamiltonian decomposition. Applications in biology' C. R. Biologies, Vol. 330 , pp. 97-106.

Gardner, M. (1970) 'Mathematical Games. The fantastic combinations of John Conway's new solitaire game «life»', Scientific American, Vol. 223, pp. 120-123.

Glade, N., Forest, L., Demongeot, J. (2007) 'Lienard systems and potentialhamiltonian decomposition. III Applications', C. R. Math., Vol. 344, pp. 253-258.

Glansdorff, P. and Prigogine, I. (1971) Structure, Stabilité et Fluctuations, Masson, Paris.

Goles E. and Salinas L. (2008) 'Comparison between parallel and serial dynamics of Boolean networks', Theoretical Computer Science, Vol. 396, pp. $247-253$.

Ishihara, S. Fujimoto, K. and and Shibata T. (2005) 'Cross talking of network motifs in gene regulation that generates temporal pulses and spatial stripes', Genes to cells, Vol. 10, pp. 1025-1038.

Kaneko, K. (2007) 'Evolution of Robustness to Noise and Mutation in Gene Expression Dynamics', PloS One, Vol. 2, e434.

Karlsson F. and Hörnquist, M (2007) 'Order or chaos in Boolean gene networks depends on the mean fraction of canalizing functions', Physica A: Statistical Mechanics and its Applications, Vol. 384, pp. 747-757.

Kauffman, S., Peterson, C., Samuelsson, B., and Troein, C. (2003)

'Random Boolean network models and the yeast transcriptional network',

Proceedings of the National Academy of Sciences of the USA, Vol. 100, pp. 14796-14799.

Kauffman, S., Peterson, C., Samuelsson, B., and Troein, C. (2004) 'Genetic networks with canalyzing Boolean rules are always stable', Proceedings of the National Academy of Sciences of the USA, Vol. 101, pp. 17102-17107. Kaufman, M., Soulé, C. and Thomas, R. (2007) 'A new necessary condition on interaction graphs for multistationarity', Journal of Theoretical Biology, Vol. 248, pp. 675-685.

Laurent, M. (1996) 'Prion diseases and the «protein only» hypothesis: a theoretical dynamic study', Biochem. J., Vol. 318, pp. 35-39.

Mendoza L. and Alvarez-Buylla, E.R. (1998) 'Dynamics of the genetic regulatory network for Arabidopsis thaliana flower morphogenesis', J. Theor. Biol., Vol. 193, pp. 307-19.

Soulé, C. (2003) 'Graphic requirements for multistability', Complexus, Vol. 1, pp. 123-133.

Thellier, M., Demongeot, J., Guespin, J., Ripoll, C., Norris, V. and Thomas, R. (2004) 'A logical (discrete) formulation model for the storage and recall of environmental signals in plants', Plant Biol., Vol. 10, pp. 1055-1075. Thom, R. (1972) Stabilité structurelle et Morphogenèse, Benjamin, New York. 